\title{
Comparative Study Between Tissues Induced Immunohistochemical Changes of Thread Granulomas and Textile Allografts
}

\author{
RADU CHICEA ${ }^{1}$, ANCA LUCIA CHICEA ${ }^{1 *}$, ALIN MIHETIU ${ }^{1}$, CRISTIAN ALEXANDRU TANTAR ${ }^{1}$, \\ MIH AELA MARIA CERNUSCA MITARIU', SEBASTIAN IOAN CERNUSCA MITARIU' ${ }^{1}$, CORINA ROMAN ${ }^{1}$, \\ RALUCA MONICA COMANEANU ${ }^{2}$, DAN BRATU', MARINELA MINODORA MANEA ${ }^{3,4}$ \\ ${ }^{1}$ Lucian Blaga University of Sibiu, Faculty of Medicine, 2A Lucian Blaga Str., 550169, Sibiu, Romania \\ ${ }^{2}$ Titu Maiorescu University of Bucharest,Faculty of Dental Medicine, 67A Gh. Petrascu Str., 031593, Bucharest, Romania \\ ${ }^{3}$ UMF Iuliu Hatieganu, 8 Victor Babes Str., 400012, Cluj Napoca, Romania \\ ${ }^{4}$ County Emergency Clinical Hospital Cluj Napoca, 3-5 Clinicilor Str., 400000, Cluj Napoca, Romania
}

\begin{abstract}
Since the time of their introduction into medical practice, biomesh were criticized for their lack of tissue integration and the rejection due to the organism reaction for an element that is not autologous. The mechanisms by which these changes of contact between the tissue and the foreign material were produced were initially incompletely understood, but over time the involvement of the immune system mediated by the cellular or humoral local inflammatory reaction was established.
\end{abstract}

Keywords: thread granuloma, biomesh, immune response.

In the beginning there was reluctance to any tissue replacement with allograft mesh, considering that an autologous material even involved in the suture is preferable to a prosthetic mesh as tissue substitution.

Tissue procedures were attributed with high tensions, poor resistance, but also with development to thread granulomas, especially in procedures in which extensive and complex sutures were required.

The mechanisms by which these changes of contact between the tissue and the foreign material were produced were initially incompletely understood, but over time the involvement of the immune system mediated by the cellular or humoral local inflammatory reaction was established [1].

Healing process involves the passage through four phases: hemostasis, inflammation, proliferation and maturation.

During hemostasis fibrin and platelet activation occurs with the progression to clot formation, followed by the inflammatory phase which involves the appearance of a mediated cellular and humoral inflammatory response.

Proliferation involves mechanisms of local angiogenesis, collagen occurrence with the appearance of an extracellular matrix and subsequent formation of granulating tissue, and for superficial structures the evolution towards epithelization [2].

The final phase of remodeling involves cellular apoptosis and alignment of collagen structures according to tension lines.

It turned out that the evolution to granuloma in a suture starts from the second phase of healing when the immune response is activated, and it persists during the three and four phases which are frequently changed by this inflammatory response.

Involvement of B and T lymphocytes in the healing / inflammation process has been proven since the 1970s by detecting their structure and with the discover of $C D 3$, CD4, CD5, CD19, CD20, CD20, CD20 components inflammation mechanisms and the inflammatory phase of healing mechanism entered a new turn [3].

CD20 is an activated glycoprotein that is present on the surface of $B$ cells. It is a natural ligand having as function to allow B lymphocyte mediated optimal immune response against T-type antigens.

Ki67 antigen is a nuclear protein involved in cell proliferation [4].

CD5 is a cluster of differentiation present in T cells (cellular mediated immunity), especially on their surface.

It is also found in lymphocytes B (humoral mediated immunity) but in a smaller amount compared to $T$ lymphocytes.

CD3 cluster differentiation $T$ cell is a receptor involved in activating the cytotoxic T cell immune response (CD8 $+)$ and Thelper immune response [2,3].

\section{Experimental part}

We compared the collagen density, fibroblast density, lymphocyte and vascularization of a biocompatible polypropylene mesh with a thread granuloma.

Paraffin embedded sections were stained for collagen with the van Gieson method using standard protocols. IHC was performed for vimentin (fibroblasts and other mesenchymal cells) CD3, CD5 and CD20 (lymphocytes), CD31 (endothelial cells), and Ki67 (cell proliferation), following the manufacturers' protocols.

Five images at $100 \mathrm{X}$ and $10 \mathrm{images} 400 \mathrm{X}$ magnifications were obtained from the proximity $(<1000$ and $200 \mu \mathrm{m}$, respectively) of the embedded material in each slide, using a Nikon Eclipse E200 microscope mounted with a Serioux cmos camera, under constant illumination settings.

Computer aided image analysis was performed in the FIJI distribution of Image], under visual control of a pathologist.

Overall collagen (100X) and collagen fiber (400X) density was measured by background substraction, followed by incremental brightness thresholding (Colour Treshold function) until all visible collagen fibers were included in the selection.

Lymphocyte density was measured by incremental brightness thresholding followed by the Analyze Particles function, with surface area and sphericity parameters set at $>100$ pixels and 50-100, respectively. 
Vimentin, CD31 and Ki67 staining was evaluated by incremental brightness thresholding and total surface measurement.

Values are expressed as mean \pm standard deviation per slide, calculated from the individual readings on each image. Statistical significance was calculated in Microsoft Excel by two tailed Student's t test. A value of $p<0.05$ was considered significant.

\section{Results and discussion}

Overall collagen density, expressed as percentage of van Gieson stained area, was $76.49 \pm 7.41 \%$ for the biocompatible mesh, significantly higher $(p<0.0001)$ compared with the granuloma (48.14 $\pm 6.84 \%$ ) (fig. 1).

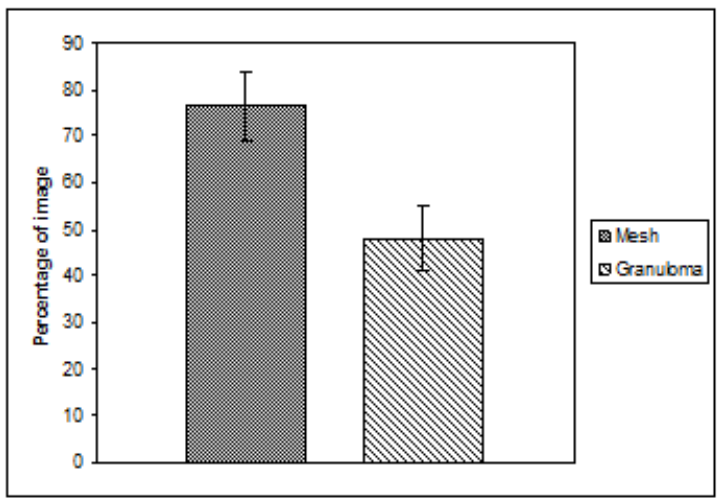

Fig. 1. Graphical representation of the comparative study between collagen density for mesh vs thread granuloma

Collagen fiber density was $50.3 \pm 9.14 \%$ for the biocompatible mesh, significantly higher $(p<0.0001)$ compared with the granuloma (21.38 $\pm 5.58 \%)$.

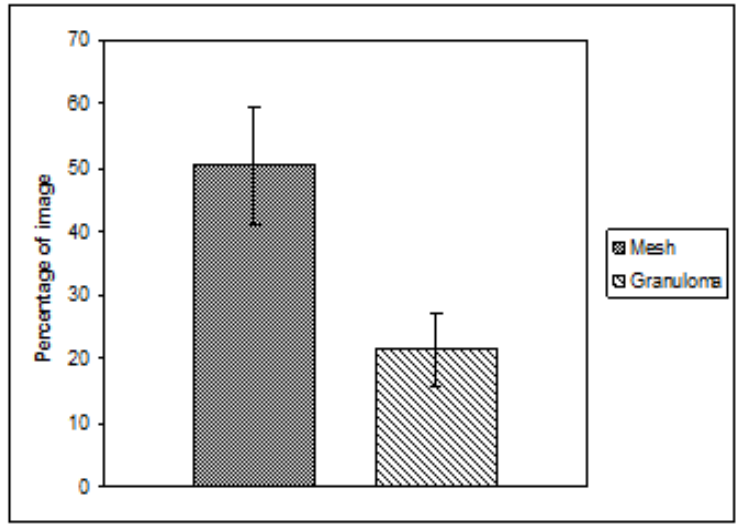

Fig. 2. Comparative representation between CD3 and CD20 frequency in mesh and thread granuloma

CD3 and CD20 positive cells were 0 negative for the biocompatible mesh, and present in the granuloma, in densities of $31.65 \pm 14.09 / 100 \mu \mathrm{m}^{2}$ and $17.6 \pm 11.25 / 100$ $\mu \mathrm{m}^{2}$, respectively.
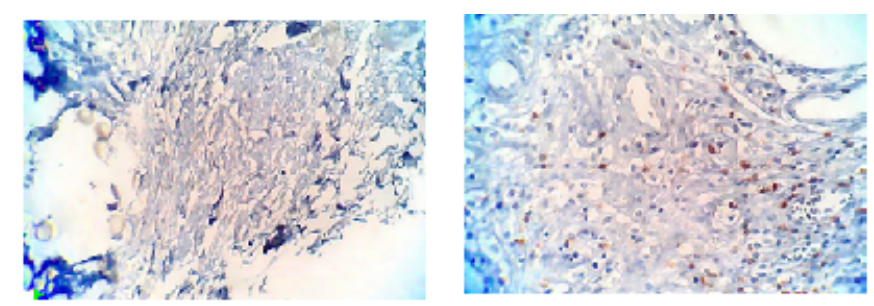

Fig. 3. Histological expression of CD3 (T cell) for biomesh and thread granuloma
CD5 positive cells were present in densities of $4.08 \pm 2.7 /$ $100 \mu \mathrm{m}^{2}$ for the biocompatible mesh, significantly lower $(p=0.002)$ compared with the granuloma ( $32.38 \pm 12.75$ / $\left.100 \mu \mathrm{m}^{2}\right)$.

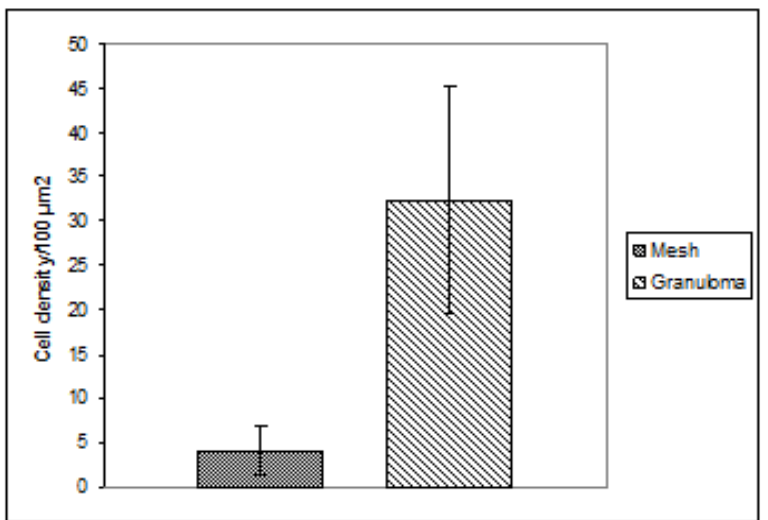

Fig. 4. Graphic comparison between CD5 density for mesh vs thread granuloma

Ki67 positive cells were 0 negative for the biocompatible mesh, and present in densities of $10.7 \pm 6.37 / 100 \mu \mathrm{m}^{2}$ in the granuloma.

CD31 staining was $7.19 \pm 4.39 \%$ for the biocompatible mesh, significantly lower $(p=0.004)$ compared with the granuloma (26.05 $\pm 8.54 \%)$.

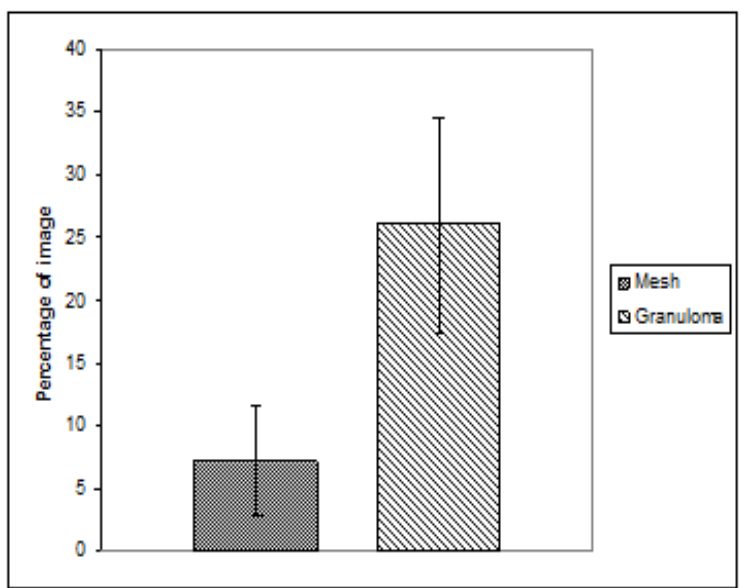

Fig. 5. Graphic comparison between CD31 staining between mesh and thread granuloma

Vimentin (VIM) staining was $12.83 \pm 3.57 \%$ for the biocompatible mesh, significantly lower $(p<0.0001)$ compared with the granuloma $(41.89 \pm 3.44 \%)$.

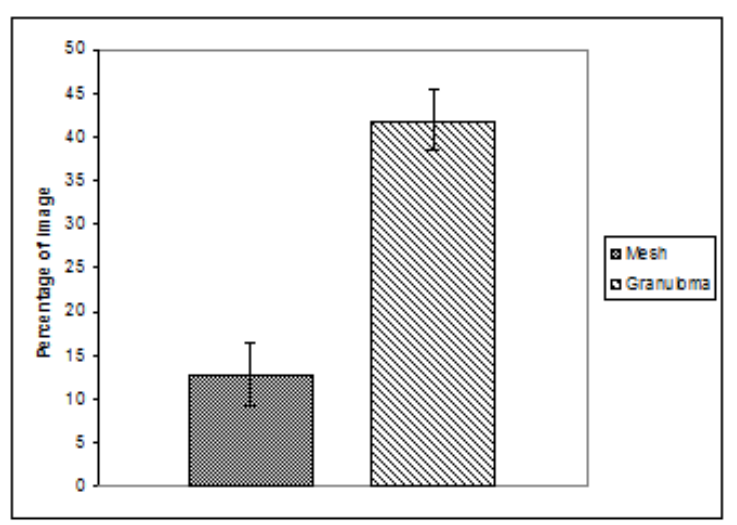

Fig. 6. Graphic comparison between Vimentin staining for mesh and thread granuloma 


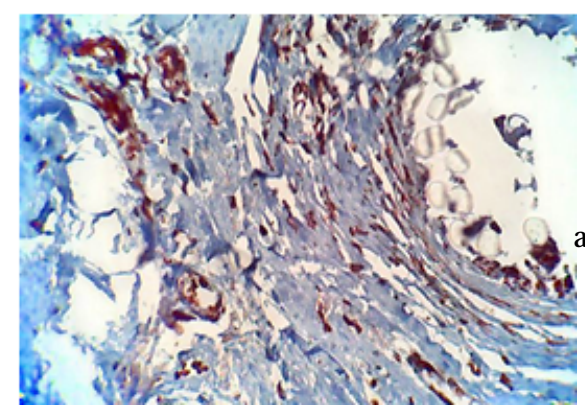

Fig. 7. Nicroscopic aspect of VIM in mesh

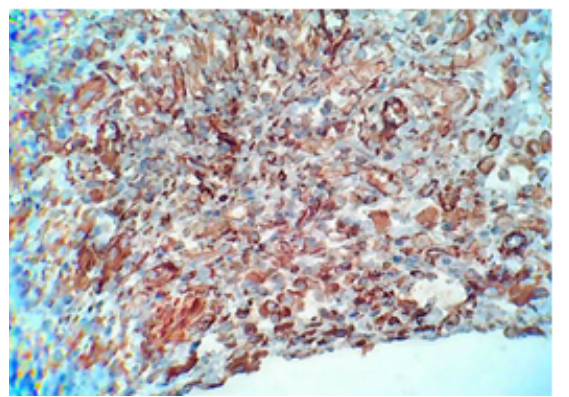

Fig. 8. Microscopic aspect of VIM in granuloma

Collagen, both in terms of quantity and density, recorded higher values in tissues which were contact with prosthetic material.

Values of the CD3 and CD20 - markers of the inflammatory phase through the cytotoxic T-cell respectively type $B$ lymphocytes immune response reveal an immune response of 0 for biomesh compared to thread granuloma.

Also, the values obtained for CD5, KI67, CD31 were smaller for mesh than for granuloma.

The study highlights a disproportionately inflammatory and immune response between the two elements: mesh and granuloma in contact with neighboring tissues. [5]

In fact, the much lower inflammatory response in the case of mesh proves the superior qualities of tissue integration that defines a good biocompatibility. A material is biocompatible when it does not harm nor create toxic reactions or systemic side effects [6].

Exacerbated inflammatory response can prolong or alter the subsequent phases of inflammation in the healing process, causing trembling inflammation, wound granulomas, scarring, rejection or local relapses [7].

On the other hand, there is a proliferation of collagen and an increase in its density in the third phase of healing, which expresses better tissue integration, higher local resistance, and due to the mesh structure and characteristics a lower tension, which prevents relapse.

\section{Conclusions}

The relationship between inflammatory response and tissue replacement material is an important component in healing quality.

The biocompatibility of tissue substitution materials recommends them to some other type of procedure.

\section{References}

1.J EFFREY M. DAVIDSON, LUISA DIPIETRO The Wound-Healing Process The Diabetic Foot, Second Edition Humana Press Inc., Totowa, NJ 2006

2.WANG PH, HUANG BS, HORNG HC, YEH CC, CHEN YJ. Wound healing. J Chin Med Assoc. 2017 Nov 21

3.KIRITSI D, NYSTROM A The role of TGFâ in wound healing pathologies Mech Ageing Dev. 2017 Nov 11

4.PATRASCU, A.M., ROTARU, I., OLARU, L, et. al.,The prognostic role of Bci-2, Ki-67, C-MYC and P53 in diffuse large B-cell lymphoma, Romanian Journal of Morphology and Embriology, vol. 58, Issue 3, pp.837-843, Published 2017

5.MARTIN-PIEDRA MA, GARZON I, GOMEZ-SOTELO A, GARCIA-ABRIL E, J AIMES-PARRABD1, LOPEZ-CANTERERO M, ALAMINOS M, CAMPOS A Generation and Evaluation of Novel Stromal Cell-Containing Tissue Engineered Artificial Stromas for the Surgical Repair of Abdominal Defects. Biotechnol J. 2017 Sep 4

6.COMANEANU, R.M., HANCU, V., BARBU, H.M., COMAN, C., COTRUT, C.M., TARCOLEA, M., HOLICOV, A.M., ORMENISAN, A., Comparative Assessment of Biocompatibility of $\mathrm{NiCr}$ and $\mathrm{CoCr}$ Alloys Used in Metal-fused-to-ceramic Technology, Rev. Chim. (Bucharest), 66, no. 3, 2015, p. 312.

7.CHICEA, R., BRATU, D., CHICEA, AL., MIHETIU, A., PRELUCA, V., TANTAR,C.,SAVA, M., A comparative Histologic and Immunohistochemistry Evaluation Between Normal Aponeurotic Tissue, Fibrotic Aponeurotic Scars and Polypropylene Embedded Aponeurotic Scars,Mat. Plast., 54, no. 3, 2017, p. 510

Manuscript received: 12.11 .2017 